\title{
Liquid Crystals as Sensitive Reporters of Lipid-Protein Interactions
}

\author{
Ipsita Pani, Diksha Sharma, and Santanu Kumar Pal* \\ Department of Chemical Sciences, Indian Institute of Science Education and Research (IISER) Mohali, \\ Sector-81, SAS Nagar, Knowledge City, Manauli-140306, India \\ Email: skpal@iisermohali.ac.in (S. K. P.)
}

\begin{abstract}
The structural integrity of biological membranes is maintained by membrane proteins embedded in the lipid bilayer. A delicate balance of weak interactions between the lipid bilayer and membrane associated proteins regulates cellular homeostasis and disease states. Recently, there has been a growing interest in the construction of in vitro mimics of biological membranes. This allows the study of multiple facets of complex interactions involving lipids and proteins in a simple environment. In recent years, liquid crystal (LC) interfaces decorated with self-assembled layers of phospholipids have evolved as biomimetic systems for systematic study of lipidprotein interactions. Binding of proteins to these phospholipid-laden fluid interfaces can be coupled to the orientational ordering of LCs. In this minireview, we have surveyed the key investigations of these interactions using LC interfaces as the sensing platform. Micrometer thick films of liquid crystals can report interactions ranging from hydrolysis of lipids by enzymatic peptides to membrane induced amyloid formation.
\end{abstract}

Keywords liquid crystals, lipids, proteins, sensors, ordering transition

\section{Introduction}

Cellular membranes are unequivocally the most important component of cells starting from primitive to the highly developed organisms. Proteins of various traits are embedded in the lipid bilayer, which is the major building block of biological membranes. An intricate balance of weak interactions between lipids, proteins and lipid-proteins imparts stability to the dynamic fabric of the biomembranes. ${ }^{[1]}$ These interactions are the chief regulators of cellular homeostasis and disease states. Over the last few decades, numerous attempts have been made to gain insights into the intervening interactions between membrane components and various peptides using high resolution spectroscopic tools. The first information about the interaction of lipids with intrinsic membrane proteins was obtained from Electron Spin Resonance (ESR) studies using nitroxide labeled phospholipid molecules. ${ }^{[2]}$ Spin labeled electron paramagnetic resonance (EPR) also provides information regarding the relative mobility of labeled acyl chains of lipids. ${ }^{[3]}$ Restricted mobility of lipid chains provides evidence for direct interactions with proteins. Another approach relies on the changes in fluorescence quenching and energy transfer of fluorescently labeled lipids and proteins as a reporter of the binding event. Cornell et al. ${ }^{[4]}$ have employed electro-analytical methods using ion channels to study interactions of proteins with bio-membranes. However, these approaches have limited applicability either due to the need for extensive labeling or complex instrumentation.

Liquid crystals (LC) can direct molecular assembly and amplify interfacial events into easily observable optical signals. The bulk orientation of LC can be easily perturbed by adsorption of polymers or amphiphiles, which can be attributed to the weak anchoring energy of the order $1-10 \mu \mathrm{J} \cdot \mathrm{m}^{-2}$. Adsorbate induced changes in LC orientation can propagate over distances of micrometers. Owing to the environment sensitive fast response of the LC, there have been increasing research efforts into utilizing LC in understanding complex biomolecular inter- actions and detection of biological analytes. Membrane-water interface covers a substantial fraction of the total membrane volume and most biological events take place in aqueous environments. LC provides an exceptional anisotropic medium to study the assembly of phospholipid monolayers. The lateral mobility of adsorbed molecules is enhanced at interfaces between nematic LCs and isotropic liquids in contrast to the solid interfaces. The aim of this minireview is to recapitulate the advances in the study of these binding events using LCs. This approach has particularly gained a lot of attention in recent years because it allows the spatial imaging of the reorganization of lipid induced protein binding events without any labeling.

\section{Biological sensing at liquid crystal interfaces}

LCs have been harnessed in many sensing applications owing to their environment sensitivity and rapid visualization of response. As pointed out by Abbott et al.. ${ }^{[5]}$ LCs possess many properties inherent to ideal biosensors. LC interfaces can be tailored to give specific response towards targeted analytes. Orientations of thermotropic nematic (rod-like) LCs are coupled to interfacial events and can be amplified easily by the transmission of polarized light. As a result, simple optical techniques such as polarizing optical microscopy (POM) can probe the orientational ordering transitions in LC. Recently, LC-based sensors have evolved as promising candidates to study biomolecular events. Many research groups have reviewed the current trends in design and development of LC-assisted sensors. ${ }^{[6-8]}$ This field has advanced in detection of chemical and biological analytes as well as understanding complex interactions involving lipids, proteins and other biomolecules.

\section{Self-assembly of lipids at LC-aqueous interfaces}

Many important biomolecular events, such as enzymatic pathways, aggregation of proteins, formation of lipid rafts, rup- 
ture of membranes by toxins, and so on, involve a reorganization of lipids and proteins. It has been demonstrated that lipidladen interfaces of LC report biomolecular interactions by adsorbate-induced ordering transitions of LCs. However, the LC response towards interfacial adsorbants is a delicate interplay of numerous parameters such as phase state, concentration, $\mathrm{pH}$, ionic strength, etc. ${ }^{[9]}$ Micrometer thick films of thermotropic nematic 5CB (4'-cyano-4-pentyl biphenyl) supported on octadecyltrichlorosilane (OTS)-coated glass slides assume homeotropic orientation (dark optical appearance) at the 5CBair interface. ${ }^{[9]}$ At lower concentrations of L-DLPC (1,2-dilauroylsn-glycero-3-phosphocholine) $(1 \mu \mathrm{M})$, the LC anchoring is similar to that in aqueous solutions (Figure 1). The tilt angle of $5 \mathrm{CB}$ increases from $0^{\circ}$ at $5 \mathrm{CB}$-OTS interface to $90^{\circ}$ at $5 \mathrm{CB}$-aqueous interface, the tilt angle of being measured with respect to the surface normal (Figure 1B). However, transfer of lipids (10-1000 $\mu \mathrm{M}_{\mathrm{L}-\mathrm{DLPC})}$ to LC-aqueous interfaces changes the optical appearance of $5 \mathrm{CB}$ from bright to dark due to perpendicular anchoring of $5 \mathrm{CB}$ at both OTS-5CB and aqueous-5CB interfaces. The perpendicular anchoring of LC upon exposure to aqueous dispersions of lipids can be attributed to the hydrophobic interactions of lipid tails with the alkyl chains of $5 \mathrm{CB} \cdot{ }^{[10]}$
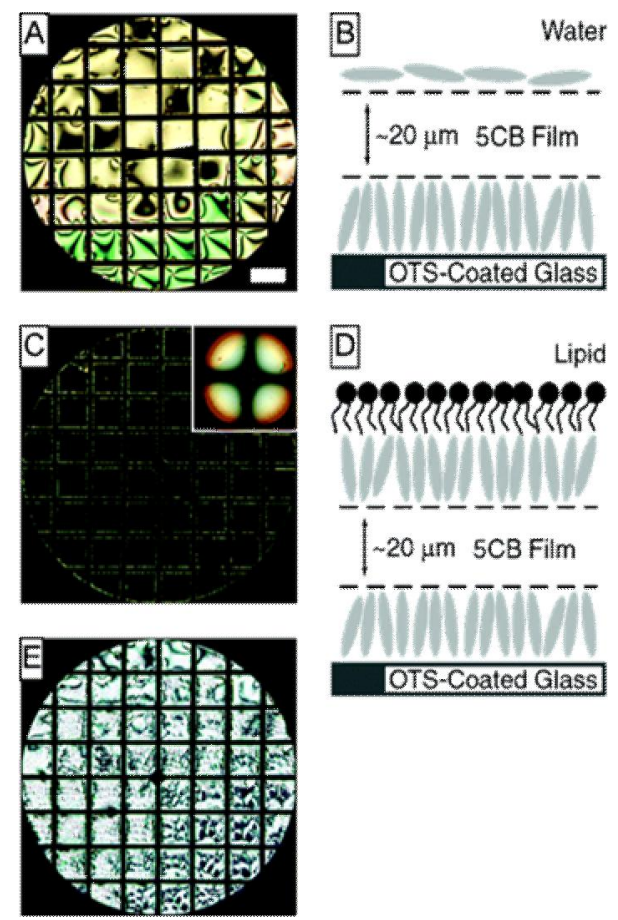

Figure 1 (A) Optical texture of 5CB after contact ( $>2 \mathrm{~h}$ ) with a dispersion of vesicles of L-DLPC in TBS at a bulk concentrations of lipid of $1 \mu \mathrm{M}$. (B) A cartoon representation of the boundary conditions within a film of $5 \mathrm{CB}$ located between the aqueous-5CB and OTS-5CB interfaces. (C) Optical texture of $5 \mathrm{CB}$ after $2 \mathrm{~h}$ of contact with a dispersion of vesicles of L-DLPC $(100 \mu \mathrm{M})$ in TBS. The inset is an interference pattern obtained by conoscopic imaging of $5 \mathrm{CB}$. (D) Cartoon representation of the uniform anchoring of the $5 \mathrm{CB}$ film after the adsorption of a monolayer of lipid at the aqueous-5CB interface. (E) Optical texture of $5 \mathrm{CB}$ after contact with a dispersion of vesicles of L-DLPC $(100 \mu \mathrm{M})$ for $10 \mathrm{~min}$. All images were obtained using crossed polars. Scale bar, $300 \mu \mathrm{m} .{ }^{[9]}$ Reproduced with permission.

Contact of L-DLPC vesicles (100 $\mu \mathrm{M}$ in TBS) with aqueous LC-interface resulted in an irreversibly adsorbed monolayer, which was confirmed by fluorescence imaging. Moreover, it was observed that lipids with low melting temperature $\left(T_{\mathrm{m}}\right)$ adsorbed readily to the interface unlike those with higher $T_{\mathrm{m}} \cdot{ }^{\left[{ }^{[0]}\right.}$ This correlates the phase state with extent of transfer of phospholipids. Another approach followed for the formation of continuous lipid layers at LC-aqueous interfaces is from mixed micelles of phospholipids and surfactants. This method permits precise control over the areal density of lipid layer assembled at the aqueous interfaces by controlling the composition of the mixed micelles. Membrane fluidity is an intrinsic property of the lipid bilayer, which plays a central role in many functions like diffusion of proteins and other bio-molecules across the membrane. ${ }^{[2]}$ Measurement of lateral diffusivity using fluorescence recovery after photobleaching (FRAP) confirmed the fluidity of L-DLPC and L-POPC (1-palmitoyl-2-oleoyl-sn-glycero-3-phosphocholine) monolayers at aqueous interfaces of LC. Thus, the observations presented above point towards the utility of biomimetic LC films for exploring interactions of biological importance.

The optical response of LC is observed as a result of coupling of lateral organization of adsorbed amphiphiles and the nematic elasticity of the LCs. ${ }^{[11]}$ In a study carried out by Gupta et al., 5CB hosted within electron microscopy grids when subjected to an aqueous solution of unilamellar vesicles of L-DLPC [doped with $0.1 \%$ Texas-Red-DPPE (1,2-dipalmitoyl-sn-glycero-3-phosphoethanolamine)] resulted in patterned orientations of $5 \mathrm{CB}$ at $32{ }^{\circ} \mathrm{C}$. These bright regions correspond to the lipid lean domains, where the LC mesogens are anchored perpendicular to the OTS-treated glass but parallel to the aqueous phase. No domain was observed above the $T_{\mathrm{NI}}$ (nematic-isotropic transition temperature) of $5 \mathrm{CB}$, which suggests that the phase separation of lipids at the interface was dictated by the nematic order of $5 \mathrm{CB}$. The authors have proposed a thermodynamic model, which relates the free energy of a lipid monolayer at LC interface with the surface anchoring energy and the elastic energy of nematic LC. In consideration of the model proposed, the phase separation of phospholipids under the experimental conditions is induced by the nematic elasticity. Secondly, thickness of LC film can influence the distribution of the adsorbed lipid molecules at the LC interface. The above model demonstrates a general mechanism for explaining the interfacial order as a function of the bulk elasticity of the nematic LC. Hence, lipid laden interfaces of LCs can be utilized for label-free protein analysis. In the next section, we will highlight the studies on lipid laden interfaces of LCs when exposed to proteins.

\section{Studies so far}

The first report, which addresses the ordering of LC in response to lateral organization of proteins on phospholipid decorated interfaces, was published by Brake et al. ${ }^{[12]}$ The interfaces between $5 \mathrm{CB}$ and aqueous phases were decorated with DPPC and DPPE (1,2-dihexadecanoyl-sn-glycero-3-phosphorethanolamine), which assumed a homeotropic anchoring of LCs pertaining to parallel orientation of the lipid tails and the LC mesogens. ${ }^{[6]}$ A specific adsorption of phospholipase $A_{2}\left(P L A_{2}\right)$ triggered a transition from homeotropic (dark appearance) to planar (bright appearance). However, no perturbation in the LC ordering was observed upon nonspecific adsorption of BSA, cytochrome-c and lysozyme. The stereospecific enzymatic hydrolysis of ${ }_{L}-D L P C$ by $P_{2} A_{2}$ induced a patterned orientation of LC driven by the reorganization of phospholipids at the interface. Additionally, the adsorption of neutravidin into mixed monolayers of L-DLPC and $\mathrm{N}$-((6-(biotinoyl)amino)hexanoyl)DPPE (Bi-X-DPPE) resulted in dendritic textures of $L C$ reporting the lateral distribution of proteins at the interface.

Additional studies have given detailed insights into the 


\section{General Chemistry}

temporal response of LC to the distribution of lipid on the aqueous interface during the enantiospecific hydrolysis reaction. ${ }^{[13]}$ The interaction of $\mathrm{PLA}_{2}$ has been investigated for three representative lipids D-DPPC (1,2-dipalmitoyl-sn-glycero3-phosphocholine), L-DPPC and L-DLPC. Unlike the L-phospholipids, $\mathrm{PLA}_{2}$ does not hydrolyze ${ }_{\mathrm{D}}$-DPPC. Further, the hydrolysis products of L-DPPC are sparingly water soluble and persist on the interface, whereas the hydrolysis products of L-DLPC are water soluble and thus desorb from the interface. Firstly, the association of $\mathrm{PLA}_{2}$ (in presence of $\mathrm{Ca}^{2+}$ ) with monolayers of D-DPPC at aqueous interfaces resulted in bright domains, which assumed a bright optical texture over time. The appearance of these domains correlated well with the spatial location of bound $\mathrm{PLA}_{2}$ at the interface when observed by epifluorescence microscopy. The bound $\mathrm{PLA}_{2}$ disturbs the lipid assembly at the interface resulting in a reorientation of the LC. In contrast to the optical response of $5 C B$ at $D$-DPPC laden interface, the specific binding of $\mathrm{PLA}_{2}$ to L-DPPC resulted in phase separated domains. Epifluorescence micrographs showed inhomogeneous distribution of the lipid reflecting the spatial localization of hydrolysis products at the interface. Lastly, examination of the dynamic response of $5 \mathrm{CB}$ to hydrolysis of L-DLPC by $\mathrm{PLA}_{2}$ at aqueous-5CB interfaces showed bright domains, which correlated to lipid depleted domains. This observation is consistent with desorption of water soluble hydrolysis products of L-DLPC. Hence, LC can report the reorganization of lipid layer by mechanisms ranging from protein association to phase separation of hydrolysis products and desorption of hydrolysis products from the interface.

In a similar report, L-DLPC decorated LC aqueous interfaces have been utilized to investigate the $\mathrm{Ca}^{2+}$ dependent phospholipase-like activity of a snake venom beta-bungarotoxin. ${ }^{[14]}$ As shown previously, the dark optical texture of $L C$ in presence of ${ }_{L}-\mathrm{DLPC}$ changed to bright as a result of enzymatic hydrolysis of the phospholipid followed by desorption of the hydrolysis products from the interface. However, no orientational transition of LC was observed upon introducing some well known phospholipase inhibitors together with beta-bungarotoxin due to the formation of toxin-substrate analogue. This work demonstrates the potential of LC-based sensors for the identification of phospholipase like toxins and screening of toxin inhibitors.

In continuation of the investigation of enzyme activity on li$\mathrm{pid} / \mathrm{LC}$ interface, Hussain et al. ${ }^{[15]}$ reported a lipase screening assay using a LC based sensor. Lipases are enzymes, which catalyze the hydrolysis of fats by breaking the ester bond. These enzymes are released after the rupture or death of a cell. In this work, the activity of lipases released from cells is measured as a direct function of change in the alignment of the LC layer coated with a membrane lipid DOPC (1,2-dioleoyl-snglycero-3-phosphocholine). When lipases from different cell lines were released onto the DOPC laden LC interface (homeotropic/dark optical appearance), a change in light transmittance was observed due to a disorder in LC alignment correlating with hydrolysis of the lipid layer. However, no change was observed upon the addition of inactivated enzymes. The LC-based sensor could detect the lipase activity of cells to a greater sensitivity using lower number of cells than the commercially available fluorescence assay.

More recently, it has also been shown that LC interfaces can be used for monitoring enzyme activity. Hu and Jang ${ }^{[16]}$ devised an approach to image enzymatic activity of trypsin at LC interfaces decorated with lipids coupled to interactions with a polyelectrolyte. A shift in the optical response of LC from dark to bright was observed when an aqueous solution of positively charged poly-L-lysine (PLL) was transferred onto the negatively charged DOPG (1,2-dioleoyl-sn-glycero-3-phosphoglycerol)
laden-5CB interface. However, when an aqueous mixture of activated trypsin and PLL were introduced to the DOPG decorated interface, the $L C$ remained dark. This approach suggests a new method for the detection of enzymatic reactions. Further, Zhang et al. ${ }^{[17]}$ have developed a simple analytical method for the detection of thrombin using LCs. Thrombin is a protease enzyme generated in the coagulation cascade and is a potential biomarker for tumor diagnosis. The optical appearance of LC changed from dark to bright when a positively charged polyelectrolyte, poly-L-arginine (PLA) was added to the negatively charged phospholipid (DOPG) monolayer on aqueous-LC interfaces. Upon addition of thrombin, which is known to hydrolyze PLA, the LCs underwent an ordering transition from planar to homeotropic state. The membrane integrity was restored as the membrane bound PLA was enzymatically cleaved by thrombin into small polyelctrolyte fragments that desorbed from the interface into the bulk solution. The detection limit of this method was found to be significantly lower than previously known methods of determination of thrombin.

In contrast to the previous reports, where proteins were adsorbed onto self assembled layers of lipids at LC interfaces, Tan et al. ${ }^{[18]}$ have shown the specific binding of phospholipid vesicles at protein decorated interfaces. LC is known to assume planar/tilted orientation at protein decorated interfaces. ${ }^{[19]}$ Specific binding of biotinylated vesicles to streptavidin decorated LC interfaces triggered a continuous ordering transition from planar to a homeotropic orientation. The dynamics of these transitions were considerably slower in case of nonspecific interactions between vesicles and proteins at interfaces (Figure 2). This difference in timescales can be attributed to the mechanism of transfer of lipids to the LC

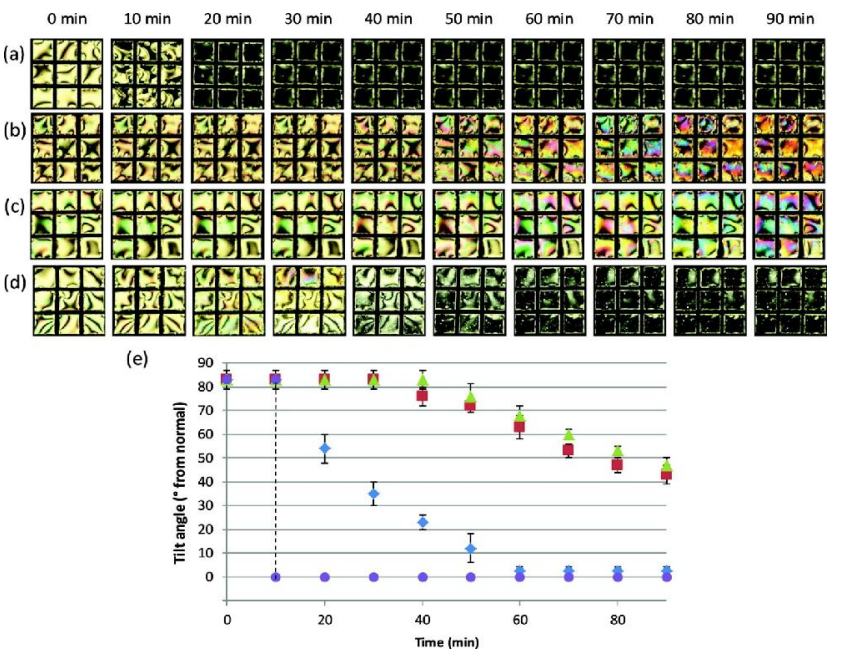

Figure 2 Ordering transitions induced in films of nematic $5 \mathrm{CB}$ by incubation of vesicles against unmodified or protein-decorated aqueous interfaces of the LC. (a) Polarized optical image of 5CB films with unmodified aqueous interface (no adsorbed protein) upon incubation against a dispersion of DLPC vesicles. (b) Optical images of 5CB with a streptavidin-decorated LC interface upon incubation against a dispersion of DLPC vesicles (no biotin-DOPE). (c) Optical images of 5CB upon incubation of a nonspecific IgG-decorated interface of the LC against a dispersion of vesicles containing biotin-DOPE. (d) Optical images of $5 \mathrm{CB}$ with a streptavidin-decorated LC interface upon incubation against a dispersion of vesicles containing 5 mol\% biotin-DOPE. (e) Tilt angle of 5CB at the aqueous-LC interface, plotted as a function of time following incubation against dispersions of vesicles [(a) circles, (b) squares, (c) triangles, and (d) diamond]. ${ }^{[18]}$ Reproduced with permission. 
interface. The specific binding of ligand functionalized vesicles to receptor decorated LC interfaces facilitates the transfer of lipids to the interface. However, the second process involves incorporation of lipids into pre-adsorbed protein films at the LC interface resulting in slower reorientation of LC. The penetration of lipids into pre-adsorbed protein layers results in slow ordering transition of LC. Hence, the time scale of LC response is found to correlate with the underlying mechanism of surface assembly.

Tercero et al. ${ }^{[20]}$ have reported a detailed study of the dynamics of protein networks at phospholipid-LC aqueous interfaces. The function of membrane proteins is found to be influenced by thickness of the surrounding phospholipid bilayer ${ }^{[1]}$ In vitro experiments have shown that the adsorption behavior of proteins is highly dependent on surface density and phase behavior of lipids at interface as mentioned by Dahmen-Levison et al. ${ }^{[20]}$ This work describes the orientational behavior of LC at sub monolayer coverage of lipid at aqueous interfaces due to penetration of proteins into the lipid layer. Non-specific adsorption of proteins on aqueous LC interfaces decorated with phospholipid monolayers could not perturb the membrane integrity. However, on introducing these proteins to an interface with sub-monolayer coverage of lipid, interesting spatial patterns were observed. Additional studies revealed that the extent of interactions between proteins and proteins-lipids dictate the spatial patterning due to penetration of the proteins into the lipid layer.

In addition, LC interfaces have also been exploited to study peptide-protein interactions. ${ }^{[21]}$ Adsorption of mixed monolayers of $\mathrm{COOH}$ and $\mathrm{OH}$ terminated lipids on the aqueous-LC interface resulted in homeotropic orientation of LC. The alignment was maintained even when NHS ( $\mathrm{N}$-hydroxysuccinimide) was added in order to activate the carboxylic acid functional groups at the interface. However, a change in optical appearance from dark to bright was observed upon conjugation of a 17-amino acid oligopeptide with activated $\mathrm{COOH}$ terminals of lipid monolayers. Interestingly, addition of enzyme trypsin, which can cleave the immobilized peptide, reverts back the LC ordering to homeotropic. This provides a facile method for real time monitoring of interfacial enzymatic interactions on peptide decorated interfaces.

Antimicrobial peptides (AMPs) are positively charged oligopeptides, which disrupt the integrity of bacterial membranes through electrostatic interactions. Hu et al. ${ }^{[22]}$ have investigated the interactions between antimicrobial agents and lipid membranes. Addition of an antimicrobial frog skin peptide PGLa to the negatively charged L-DOPG led to the reorganization of DOPG monolayer, whereas the zwitter-ionic DOPC (1,2dioleoyl-sn-glycero-3-phosphocholine) monolayer remained intact. These results show an easy way to study the mechanism of microbe-assisted membrane permeabilization induced by antimicrobial agents.

Lipid laden films of LC provide a simple analytical tool for understanding biologically significant interactions with proteins. Quantification of LC response towards these interfacial interactions can provide estimates of relative binding affinities. Das et al. ${ }^{[23]}$ have found that honey bee apitoxin (melittin) upon interaction with DLPC induces ordering transitions in LC at aqueous interfaces. Melittin is known to permeate into lipid bilayers of mammalian cell membrane thus helping in the passage of bee venom into blood serum. As melittin has high affinity towards $\mathrm{Ca}^{2+}$, faster response time and greater sensitivity were observed when incubated with $\mathrm{Ca}^{2+}$. Furthermore, conformational studies of melittin carried out using circular dichroism (CD) and vibrational circular dichroism (VCD) in solution revealed that melittin has alpha helical conformation. A

decrease in ellipticity was observed in the presence of $\mathrm{Ca}^{2+}$ thereby making it more exposed to interact with DLPC. However, in presence of DLPC, melittin adopts a more ordered conformation. The spectroscopic read outs corroborate with the orientational response of LC towards thermodynamically driven binding of honey bee venom with DLPC. Hence, this report demonstrates a simple optical method to quantify mellitinphospholipid interactions.

Bacterial endotoxin or lipopolysachharide (LPS) is involved in many pathological infections. The extent of interactions of LPS with different proteins of the body fluid has different effects on its endotoxicity. LPS, being an amphiphilic molecule with a polar head group and long alkyl chains, changed the optical appearance of the aqueous-LC interface from bright to dark due to the coupling between the aliphatic chains of the adsorbed LPS and the LCs. ${ }^{[24]}$ This paper advances towards the study of LC response at LPS laden aqueous interfaces in presence of three representative proteins haemoglobin $(\mathrm{Hb})$, lysozyme (LZM) and bovine serum albumin (BSA), which are known to have divergent interactions with LPS. Introduction of all three proteins changed the orientation of LC from homeotropic to planar/tilted. Since these proteins have different charges at physiological $\mathrm{pH}$, the ordering of LC was found to be independent of electrostatic interactions. Moreover, the measured gray scale intensity was found to correlate with the concentrations and the affinity constant values of LPS-protein binding (Figure 3). The magnitude of binding constants follows the

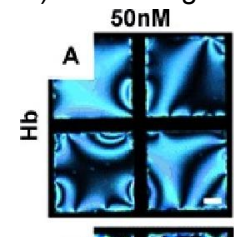

$10 \mathrm{nM}$
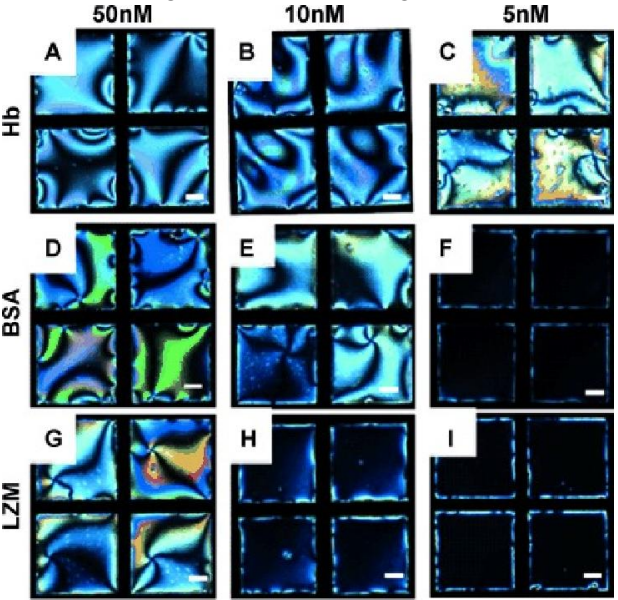

(J)

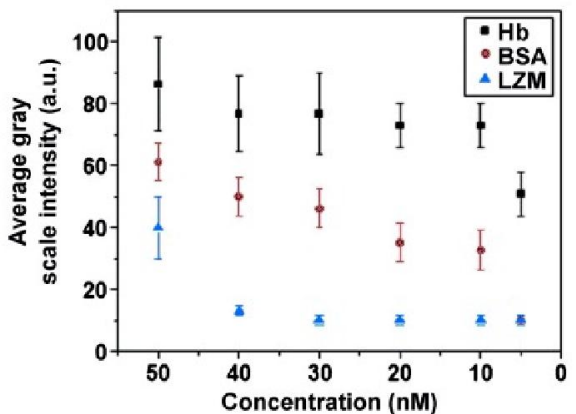

Figure 3 Polarizing optical micrographs of 5CB contained in gold grids supported on DMOAP-treated glass slides and placed in contact with the LPS-laden aqueous interface followed by incubation for 60 min with $50(A-C), 10(D-F)$, and 5 $\mathrm{nm}(\mathrm{G}-\mathrm{I})$ aqueous solutions of $\mathrm{Hb}(\mathrm{A}, \mathrm{D}, \mathrm{G})$, BSA $(B, E, H)$, and LZM (C, F, I). J) Average grayscale intensity of optical images of $5 \mathrm{CB}$ films as a function of varying concentrations of $\mathrm{Hb}$, BSA, and LZM on the LPS-decorated aqueous/5CB interface. Scale bar $=40 \mu \mathrm{m} .{ }^{[24]}$ Reproduced with permission. 
order: Hb-LPS > BSA-LPS > LZM-LPS. A gradual change in the tilt angle was observed due to the binding of proteins to the LPS-decorated LC-interfaces. Similar trend in binding affinity was revealed when tilt angles of LC were measured. This offers a simple analytical tool to quantify LPS-protein interactions at aqueous interfaces of $L C$.

Hartono and coworkers ${ }^{[25]}$ have reported that the direct interaction of AuNPs with L-DLPC monolayer at aqueous LC interfaces could not affect its integrity, whereas AuNPs coated with proteins were found to disrupt the monolayer leading to the bright optical appearance of LC. In addition, no disruption in the monolayer was observed in the case of free proteins. The reason for change in the optical appearance might be attributed to conformational changes in proteins after being coated on the AuNP surface or due to multiple proteins adsorbed on AuNP surface interacting simultaneously with L-DLPC. Furthermore, it was observed that larger the size of the protein coated nanoparticles, more was the extent of disruption of lipid layer. The authors interpret that the hydrophobic interactions between the protein-coated nanoparticles and phospholipid monolayer plays a major role in the disruption of the L-DLPC monolayer. The study offers a platform to investigate physical interactions between nanoparticles and phospholipid membrane, which is pivotal for the understanding of cytotoxicity of nanomaterials.

An interesting attribute of the LC-based sensing was revealed by Sadati et al., ${ }^{[26]}$ where they have shown that LCs can give specific response towards $\beta$-sheet rich amyloid fibrils of peptides like amyloid $\beta$, hIAPP and Q16 in the presence of lipid monolayers. In contrast to proteins, which exhibit $\alpha$ helical conformations, these proteins form elongated branched structures at the interface (Figure 4). These domains correspond to the formation of amyloid fibrils as confirmed by fluorescence experiments with amyloid marker Thioflavin T (ThT). (Figure 4g) Theoretical computations show that amyloid fibrils exhibit a stronger anchoring energy of magnitude $10^{-3} \mathrm{~J} \cdot \mathrm{m}^{-2}$ in comparison to the weaker anchoring energy of $10^{-4} \mathrm{~J} \cdot \mathrm{m}^{-2}$ shown by $\alpha$ helical proteins. This property of LC could be leveraged in the detection of $\beta$-sheet proteins, which are generally involved in neurological disorders at early stages.

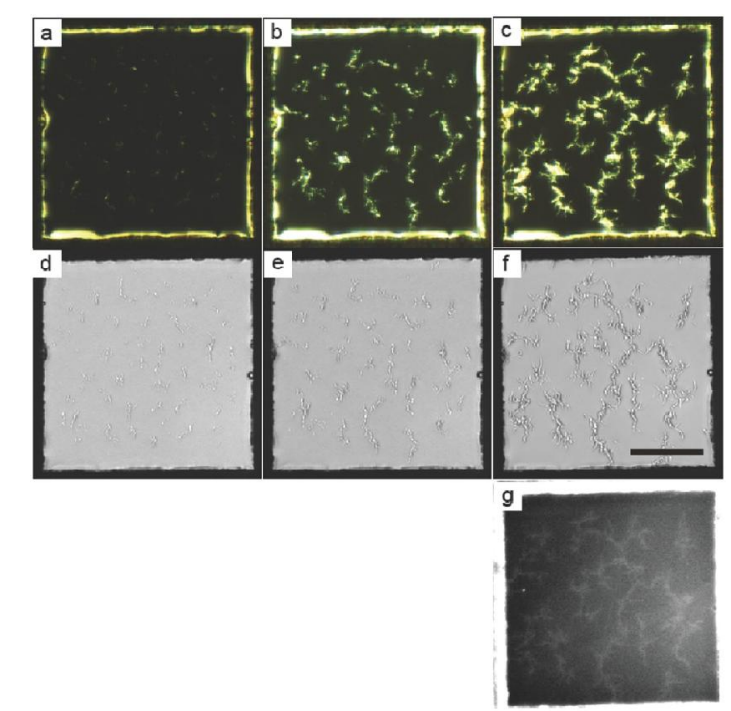

Figure 4 Dynamic optical response of the lipid-laden interface of LC to hIAPP adsorption. (a-c) Polarized light images and (d-f) images obtained by removal of the analyzer. $(g)$ The epifluorescence image of ThT fluorescence. The images taken at (a, d) 3 (h, b, e) $6 \mathrm{~h}$, and (c, f, g) $18 \mathrm{~h}$ after injection of the peptide into the aqueous phase. The scale bar is $100 \mu \mathrm{m} .{ }^{[26]}$ Reproduced with permission.

\section{Conclusions}

Many biological events of primary importance, such as protein folding and aggregation, enzymatic reactions, microbial infection, occur at interfaces of cellular membranes. ${ }^{[7]}$ LC aqueous interfaces are excellent biomimetic systems as they offer a unique combination of fluidity and allow the study of biological phenomena at aqueous interfaces. In this minireview, we have outlined the principles and recent investigations on LC based sensing of biomolecular interactions. The mechanisms underlying the change in anchoring of LCs at phospholipid decorated interfaces range from reorganization of lipids due to penetration of proteins to enzymatic hydrolysis of the lipid layer and subsequent desorption of products from the interface. LCs also respond to the membrane induced aggregation of amyloidogenic proteins, which can have serious implications in various neuro-pathologies. Thus, the panoply of applications of LC-based sensing has placed the field in the context of emerging trends to study complex biomolecular interactions in a simple environment.

\section{Acknowledgement}

This work was carried out with the financial support from IISER Mohali. I. Pani acknowledges the receipt of a graduate fellowship from IISER Mohali. D. Sharma acknowledges the INSPIRE fellowship. Dr. S. K. Pal is grateful for INSA Medal for Young Scientist 2015 and the financial support from INSA bearing sanction No. SP/YSP/124/2015/433. All the figures are reproduced with permission from respective publishers and acknowledged.

\section{Author Contributions}

The manuscript was written through contributions of all authors. All authors have given approval to the final version of the manuscript.

\section{Special Issue}

This paper is dedicated to the special issue of "Liquid Crystals".

\section{References}

[1] Tamm, L. K. Protein-Lipid Interactions: From Membrane Domains to Cellular Networks, Wiley-VCH, Weinheim, Germany, 2005.

[2] Lee, A. G. Biochim. Biophys. Acta 2003, 1612, 1.

[3] Battle, A. R.; Ridone, P.; Bavi, N.; Nakayama, Y.; Nikolaev, Y. A.; Martinac, B. Biochim. Biophys. Acta 2015, 1848, 1744.

[4] Cornell, B. A.; Braach-Maksvytis, V. L. B.; King, L. G.; Osman, P. D. J.; Raguse, B.; Wieczorek, L.; Pace, R. J. Nature 1997, 387, 580.

[5] Gupta, V. K.; Skaife, J. J.; Dubrovsky, T. B.; Abbott, N. L. Science 1998, 279, 2077.

[6] Carlton, J. C.; Hunter, J. T.; Miller, D. S.; Abbasi, R.; Mushenheim, P. C.; Tan, L. N.; Abbott, N. L. Liq. Cryst. Rev. 2013, 1, 29.

[7] Popov, N.; Honaker, L. W.; Popova, M.; Usol'tseva, N.; Mann, E. K.; Jákli, A.; Popov, P. Materials 2018, 11, 20.

[8] Setia, S.; Sidiq, S.; De, J.; Pani, I.; Pal, S. K. Liq. Cryst. 2016, 43, 2009.

[9] Brake, J. M.; Daschner, M. K.; Abbott, N. L. Langmuir 2005, 21, 2218.

[10] Lockwood, N. A.; Abbott N. L. Curr. Opin. Colloid Interface Sci. 2005, 10, 111.

[11] Gupta, J. K.; Meli, M.-V.; Teren, S.; Abbott, N. L. Phys. Rev. Lett. 2007, 100, 048301. 
[12] Brake, J. M.; Daschner, M. K.; Luk, Y. Y.; Abbott, N. L. Science 2003, 302, 2094.

[13] Brake, J. M.; Abbott, N. L. Langmuir 2007, 23, 8497.

[14] Hartono, D.; Lai, S. L.; Yang, K-L.; Yung, L-Y. L. Biosens. Bioelectron. 2009, 24, 2289.

[15] Hussain, Z.; Zafiu, C.; Küpcü, S.; Pivetta, L.; Hollfelder, N.; Masutani, A.; Kilickiran, P.; Sinner, E. K. Biosens. Bioelectron. 2014, 56, 210

[16] Hu, Q. Z.; Jang, C. H. ACS Appl. Mater. Interfaces 2012, 4, 1791.

[17] Zhang, M.; Jang, C. H. Anal. Biochem. 2014, 455, 13.

[18] Tan, L. N.; Orler, V. J..; Abbott, N. L. Langmuir 2012, 28, 6364.

[19] Tingey, M. L.; Wilyana, S.; Snodgrass, E. J.; Abbott, N. L. Langmuir 2004, 20, 6818.

[20] Tercero, M. D. D.; Abbott, N. L. Chem. Eng. Commun. 2009, 196, 234

[21] Park, J. S.; Abbott, N. L. Adv. Mater. 2008, 20, 1185.
[22] Hu, Q. Z.; Jang, C. H. Analyst 2012, 137, 567.

[23] Das, D.; Pal, S. K. ChemistrySelect 2017, 2, 4779.

[24] Das, D.; Sidiq, S.; Pal, S. K. ChemPhysChem 2015, 16, 753

[25] Hartono, D.; Qin, W. J.; Yang, K. L.; Yung, L. Y. L. Biomaterials 2009, 30, 843 .

[26] Sadati, M.; Lai, Apik, A. I.; Perez, J. C. A.; Gonzalez, J. M.; Ortiz, J. P. H.; Abbott, N. L.; de Pablo, J. J. Adv. Funct. Mater. 2015, 25, 6050.

[27] Findlay, H. E.; Booth, P. J. J. Phys.: Condens. Matter. 2006, 18, 28.

Received April 7, 2018

Accepted May 3, 2018 